\title{
Patient selection essential in optimizing the benefit of radical prostatectomy for patients with organ-confined prostate cancer
}

\author{
Matthew J Resnick and Thomas J Guzzo \\ Asian Journal of Andrology (2011) 13, 789-790; doi:10.1038/aja.2011.124; Published online: 12 September 2011
}

$\mathrm{P}$ rostate cancer is the most common noncutaneous malignancy in the United States and is a highly prevalent disease worldwide in industrialized nations. ${ }^{1}$ Prostate cancer is also a leading cause of cancer related mortality in the United States with an estimated 32050 deaths in $2010 .^{1}$ If one were to simply look at the raw mortality data, treatment decisions would appear to be cut and dry; however, they are anything but. The era of prostate-specific antigen (PSA) screening has led not only to an increase in the detection of prostate cancer, but also a substantial stage migration favoring low-volume, low-grade disease. $^{2}$ Given the number of treatment options available for patients with clinically localized prostate cancer, the water is murky at best both for clinicians and patients attempting to make appropriate treatment decisions.

There are currently more questions than answers for patients with clinically localized prostate cancer. It is well established that radical prostatectomy provides excellent cancer control in properly selected men with prostate cancer. ${ }^{3}$ Yet, despite our greater understanding of pelvic anatomy and improvements in surgical technique, decrements in quality of life following radical prostatectomy continue to be significant when assessed by validated, patientreported standards. ${ }^{4}$ It is therefore paramount to distinguish which patients will ultimately benefit from surgery, while sparing those who are at low risk for prostate cancer-related mortality the morbidity of such treatment. The current problem lies in distinguishing these two groups of patients.

The Division of Urology, The Hospital of the University of Pennsylvania, Philadelphia, PA 19104, USA Correspondence: Dr TJ Guzzo (thomas.guzzo@ uphsupenn.edu)
The Scandinavian Prostate Cancer Group Study 4 (SPCG-4) recently reported followup data from their study comparing radical prostatectomy and watchful waiting. ${ }^{5}$ This study randomized 695 men with prostate cancer to either radical prostatectomy or watchful waiting from October 1989 to February 1999. The authors have now reported estimated 15 year results, with a median follow-up of 12.8 years. Subgroup analysis was also reported according to age $(<65$ years $v s . \geqslant 65$ years), PSA at the time of diagnosis $\left(<10 \mathrm{ng} \mathrm{ml}^{-1} v s\right.$. $\left.\geqslant 10 \mathrm{ng} \mathrm{ml}^{-1}\right)$, Gleason score $(<7 v s$. $\geqslant 7)$ and also in low risk (Gleason score, $<7$; PSA, $<10 \mathrm{ng} \mathrm{ml}^{-1}$ ) patients. The updated analysis again revealed that those undergoing radical prostatectomy demonstrated improved overall survival (6.6\% absolute difference), lower risk of death from prostate cancer $(6.1 \%$ absolute difference) and a lower risk of distant metastasis ( $11.7 \%$ absolute difference) than those randomized to watchful waiting. When stratified by age, men of $\geqslant 65$ years did not benefit from radical prostatectomy for any of the three primary outcomes studied. Additionally, although men of $\leqslant 65$ years with low risk disease who were randomized to radical prostatectomy did enjoy improvements in overall survival (19.3\%), death from prostate cancer $(4.5 \%)$ and distant metastasis $(11.1 \%)$; only the difference in overall survival achieved statistical significance (0.002).

The SPCG-4 randomized trial, now with long-term follow-up, provides valuable insight for practitioners who treat prostate cancer; however, these observations must be viewed within the context of this study population. First, this is not a PSA screen-detected prostate cancer population and therefore, this cohort is not comprised of the low-risk patients found in a typical contemporary screen-detected population. ${ }^{6}$ Second, nearly half of the patients who underwent radical prostatectomy in the SPCG-4 were found to have extracapsular disease on final pathologic review, again not reflecting contemporary cohorts of radical prostatectomy patients Finally, the subgroup analysis was not intended in the initial randomized trial protocol and therefore, conclusions based on this data must be interpreted cautiously.

Radical prostatectomy is the gold standard treatment for prostate cancer in men with a life expectancy $>10$ years and is the benchmark by which all other treatment modalities should be measured. In the most recent analysis by Bill-Axelson et al., ${ }^{5}$ the number of radical prostatectomies needed to prevent one death in the entire cohort was 15 . The number needed to treat was even lower (7) for men at $<65$ years of age. While the number needed to treat for men $<65$ years in the SPCG-4 study is quite acceptable, it has been less flattering in recent prostate cancer screening trials including the ERSPC study in which the number needed to treat to prevent one prostate cancer death was 48 at 9 years of follow-up. ${ }^{7}$ It is likely that the number needed to treat in trials such as the ERSPC will continue to decline with longer follow-up; however, these data do underscore the dilemma of prostate cancer over diagnosis and over treatment in a screen-detected population. While radical prostatectomy does save lives, the 'one size fits all' approach to surgical treatment is not an effective management strategy in the screen-detected population with regard to risk of death from prostate cancer, surgical morbidity, health care cost and resource allocation. Radical prostatectomy certainly has an important role in the treatment of prostate cancer; however, we as clinicians are charged with delivering therapy to those most 
at need while minimizing the burden of treatment in those who are at low risk of disease progression and prostate cancer-related death. While an optimal strategy has yet to be defined, the SPCG-4 trial does imply that men of $\geqslant 65$ years who are diagnosed with prostate cancer may be ideal candidates for active surveillance. Additionally, it calls into question the utility of mass PSA screening in older males, an approach which has already been discouraged by the US Preventative Services Task Force. ${ }^{8}$

The field of prostate cancer is sorely in need of additional prognostic tools in the form of more sensitive and specific biomarkers in combination with advanced radiographic techniques to identify tumors with aggressive biological potential that can be differentiated from more indolent tumors. With a better understanding of tumor biology, radical prostatectomy can be offered to men who will benefit from surgical resection of their disease, while confidently avoiding the cost and morbidity of unnecessary treatment in those who will not benefit from it.

1 Jemal A, Siegel R, Xu J, Ward E. Cancer statistics, 2010. CA Cancer J Clin 2010; 60: 277-300.

2 Strope SA, Andriole GL. Prostate cancer screening: current status and future perspectives. Nat Rev Urol 2010; 7: 487-93.

3 Eggener SE, Scardino PT, Walsh PC, Han M, Partin AW et al. Predicting 15-year prostate cancer specific mortality after radical prostatectomy. J Urol 2011; 185: 869-75.

4 Xylinas E, Ploussard G, Durand X, de La Taille A, Gillion $\mathrm{N}$ et al. Oncological and functional outcomes after radical prostatectomy: trifecta rate of achieving continence, potency and cancer control-a literature review. Urology 2010; 76: 1194-8.

5 Bill-Axelson A, Holmberg L, Ruutu M, Garmo H, Stark JR et al. Radical prostatectomy versus watchful waiting in early prostate cancer. N Engl J Med 2011; 364: 1708-17.

6 Andriole GL, Crawford ED, Grubb RL 3rd, Buys SS, Chia $D$ et al. Mortality results from a randomized prostate-cancer screening trial. N Engl J Med 2009; 360: 1310-9.

7 Schröder FH, Hugosson J, Roobol MJ, Tammela TL, Ciatto $S$ et al. Screening and prostate-cancer mortality in a randomized European study. N Engl J Med 2009; 360: 1320-8.

8 US Preventive Services Task Force. Screening for prostate cancer: U.S. Preventive Services Task Force recommendation statement. Ann Intern Med 2008; 149: 185-91. 